\title{
Femtosecond laser fabrication of optofluidic devices for single cell manipulation
}

\author{
Francesca Bragheri ${ }^{1, a}$, Rebeca Martinez Vazquez ${ }^{1}$, Petra Paiè ${ }^{2}$, Tie Yang $^{3}$, Paolo Minzioni ${ }^{3}$, Ilaria Cristiani ${ }^{3}$, Roberto \\ Osellame $^{1,2}$ \\ ${ }^{1}$ Istituto di Fotonica e Nanotecnologie, CNR, Piazza Leonardo da Vinci 32, 20133 Milano, Italy \\ ${ }^{2}$ Dipartimento di Fisica, Politecnico di Milano, Piazza Leonardo da Vinci 32, 20133 Milano, Italy \\ ${ }^{3}$ Dept. of Electrical, Computer, and Biomedical Engineering, Università di Pavia, Via Ferrata 5A, 27100 Pavia, Italy.
}

\begin{abstract}
In this work we fabricate and validate two optofludic devices for the manipulation and analysis of single cells. The chips are fabricated by femtosecond laser micromachining exploiting the $3 \mathrm{D}$ capabilities of the technique and the inherent perfect alignment between microfluidic channels and optical networks. Both devices have been validated by probing the mechanical properties of different cancer cell lines, which are expected to show different elasticity because of their different metastatic potential.
\end{abstract}

\section{Introduction}

Cell mechanics plays a very important role in various cellular functions and in many disease-related mutations, especially for carcinogenesis [1]. Indeed, many studies revealed a clear correlation between cellular transformations and changes in its mechanical properties [2]. Recently the development of integrated and low-cost optofluidic devices able to handle single cells allowed probing mechanical properties of cells in a non-invasive a manner [3-5]. Such devices rely on microfluidic circuits that guarantee a controlled flow of the sample and optical radiations are often exploited to probe or manipulate the cells under test. Among the different microfabrication technologies, femtosecond laser micromachining (FLM) [6] is ideally suited for this purpose as it provides the integration of both microfluidic and optical functions on the same glass chip leading to monolithic, perfectly aligned, robust and portable optofluidic devices. The main advantages are rapid prototyping, three-dimensional capabilities and inherent alignment precision between optical and fluidic components. Here we present two recently developed integrated optofluidic microchips fabricated by $3 \mathrm{D}$ femtosecond laser micromachining to analyze cell mechanics. In the first chip (an optical stretcher and sorter, OS in the following) optical forces are used both to induce cell deformation and to sort the cells, whereas in the second one (a constriction-chip, CC in the following) mechanical properties are tested in terms of cells' ability to passively squeeze through a small constriction $[7,8]$.

\footnotetext{
${ }^{a}$ francesca.bragheri@ifn.cnr.it
}

\section{Fabrication of the devices}

Femtosecond laser micromachining is exploited to fabricate both the optical and the fluidic components of the devices. The technique is a two-step fabrication process consisting of: 1) permanent material modification due to nonlinear absorption of focused femtosecond laser pulses; 2) etching of the laser modified zone by a hydrofluoric acid (HF) solution. The laser irradiation enhances the etching rate by up to two orders of magnitude with respect to the pristine material, enabling the manufacturing of channels with arbitrary shape in the bulk glass substrate.

The irradiation is performed by focusing through a $50 \mathrm{x}$, 0.6 NA microscope objective the second harmonic of a commercial femtosecond laser (femtoREGEN, HIGH-Q Laser) emitting pulses of $400 \mathrm{fs}, 1040 \mathrm{~nm}$ wavelength and energy up to $23 \mu \mathrm{J}$ at $960 \mathrm{kHz}$ repetition rate. Scan velocities and pulse energies are varied in relation to the type of the irradiated structure (e.g. waveguides or microchannels). The geometry of the devices is obtained by properly moving the sample with respect to the laser beam with a system of high precision air bearing translation stages (Fiberglide 3D, Aereotech). The detailed fabrication process is described in the following sections for the two different devices.

\subsection{Optical stretcher and sorter}

The geometry of the device is similar to the one already reported in the literature [9], where the channel roughness remained an issue since it hindered a good image quality.

This is an Open Access article distributed under the terms of the Creative Commons Attribution License 4.0, which permits unrestricted use, distribution, and reproduction in any medium, provided the original work is properly cited. 
In order to overcome this limitation we fabricate the microfluidic channels parallel to the writing beam by irradiating them from the bottom to the top of the substrate, as schematically shown in Fig. 1.
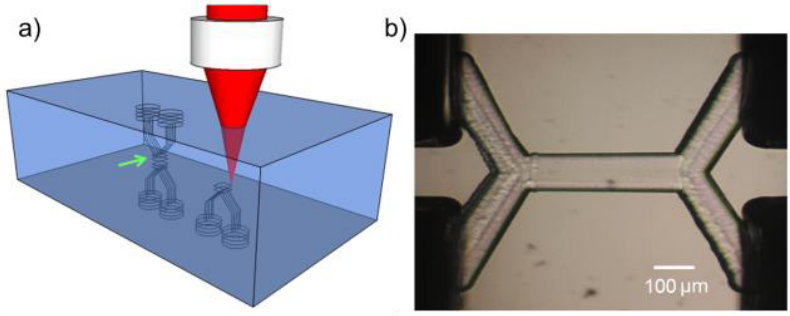

Figure 1. a) Schematic of the irradiation configuration and pattern. b) Picture of the device after the etching process.

The central channel, with a square cross-section, is obtained by irradiating overlapped squares at different depths. The laser power and scanning velocity are varied along the substrate depth to overcome the effects of spherical aberrations: the irradiation is performed with an energy of $400 \mathrm{~nJ}$ and a translation speed of $0.07 \mathrm{~mm} / \mathrm{s}$ for the bottom Y-branch, $300 \mathrm{~nJ}$ and $0.1 \mathrm{~mm} / \mathrm{s}$ for the central square channel, $300 \mathrm{~nJ}$ and $0.2 \mathrm{~mm} / \mathrm{s}$ for the top Ybranch. To produce the central square channel we irradiated a pattern of $60 \mu \mathrm{m}$ squares at different depths (with a separation of $2 \mu \mathrm{m}$ over a total length of $300 \mu \mathrm{m}$ ). Vertical lines are also irradiated inside the squares to facilitate glass removal by the acid. The Y-shaped branches are obtained by multiscan irradiation of 10 lines in order to obtain, after etching, channel with the same height as the central one. Access holes for the tubing connection are realized by irradiating 4 coaxial circular helixes at each termination of the Y-branches. The irradiation pattern is shown in Fig. 1(a). In the same irradiation step we also fabricated the optical waveguides for the stretching and sorting functionalities by using pulses of $60 \mathrm{~nJ}$ energy and a scan speed of $0.01 \mathrm{~mm} / \mathrm{s}$. After irradiation, the glass substrate is immersed in a $20 \%$ aqueous solution of hydrofluoric acid at $35^{\circ} \mathrm{C}$ in an ultrasonic bath for 1.5 hours. The final result is shown in Fig. 1(b).

\subsection{Constriction chip}

Again the optofluidic chip was entirely fabricated by FLM. In particular, the microfluidic network was created by fs laser irradiation followed by chemical etching (FLICE) and the waveguide just by fs laser irradiation, with parameters previously reported [9]. The complete device obtained after the irradiation and etching procedure is shown in Fig. 2a). A detail of the irradiation for the fabrication of the constriction section is shown in Fig. 2b), an irradiated region is embedded between two non-irradiated ones that will afterwards constitute the constriction region. These non-irradiated regions slow down the etching process, thus exposing the constriction region for a much shorter etching time. This results in a $3 \mathrm{D}$ elliptical section constriction with axial dimensions of $7 \times 30 \mu \mathrm{m}(\mathrm{y} \times \mathrm{z})$ and a length (x direction) of $15 \mu \mathrm{m}$.

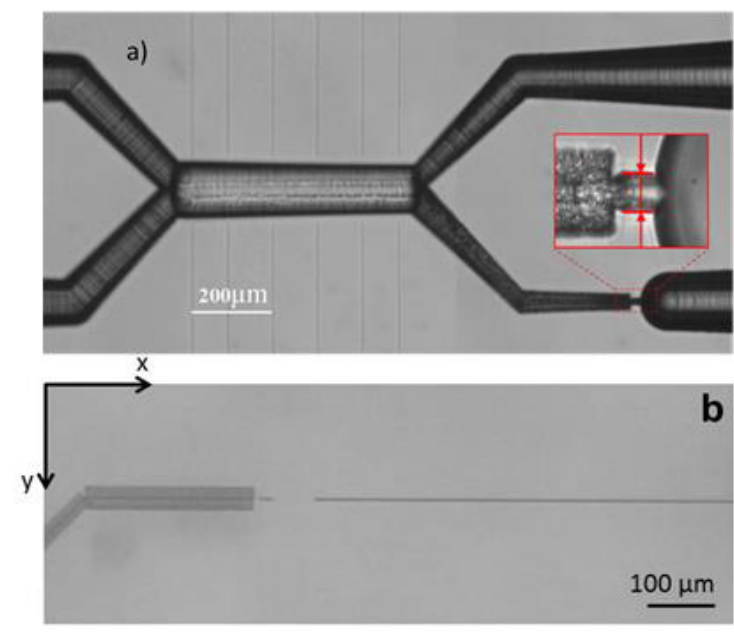

Figure 2. a) Bright field microscope image of the constriction chip with local enlarged constriction part: $8 \times 12 \mu \mathrm{m}^{2}$. b) Microscopy image of the irradiation pattern at the constriction region.

\section{Mechanical properties results}

Both devices have been validated by probing mechanical properties of two cell lines, that are expected to show different elasticity because of their different metastatic potential, while keeping the system temperature constant so as to avoid temperature induced variations of cells properties [10].

\subsection{OS measurement procedure and results}

After the chip fabrication and pigtailing, the device is connected to a 10-W fiber laser (emitting@1070 nm), and to tubings (see setup in Fig.3a).

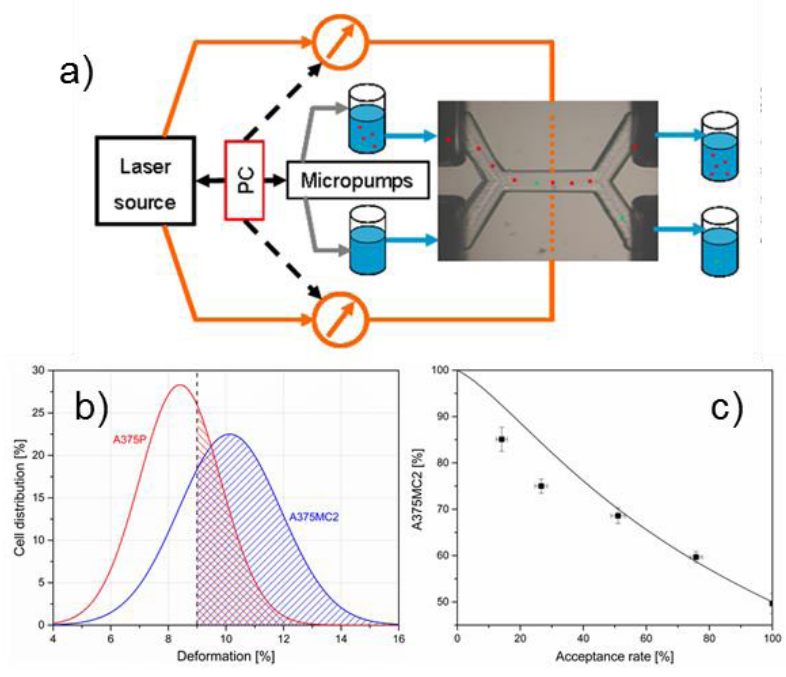

Figure 3. a) Scheme of the setup used for the experiment. b) Optical deformation distribution curve for A375-P and A375MC2 cells. The highlighted area show which is the portion of the two populations selected when the deformation threshold is set at $9 \%$. c) Curve showing the correspondence between changes in the concentration of A375-MC2 cells in the output sample and changes in the "acceptance rate" when the threshold is raised from $0 \%$ to $20 \%$. 
System validation is obtained by injecting at one of the input a buffer solution, while at the other a suspension containing two different cellular lines of human melanoma (metastatic A375-P, and highly-metastatic A365-MC2). In the starting suspension the ratio of the cells belonging to the two lines is $1: 1$. Thanks to the fact that the two lines show the same cellular size, but a slightly different optical deformability (see Fig.3b) we aimed at selecting a population of cells with an enriched content of A375-MC2 cells. To achieve this result we defined a "deformation threshold" (e.g. 9\%, as shown in Fig.3b) and we sorted to a "selected-cells" vial only those cells showing a deformation higher than the threshold. By repeatedly performing "stretching \& sorting" tests, also considering different "deformation thresholds", we verified that both the experimentally obtained enrichment-factor and the cells "acceptance rate" (i.e. the percentage of cells in the input sample exhibiting a deformability higher than the threshold) closely match the theoretically expected values, thus demonstrating the efficiency and reliability of the proposed chip.

\subsection{CC measurement procedure and results}

The measurement principle is simple: at the two inlets, on the left-end of Fig 2a, we input a cell suspension and a buffer fluid, top and bottom input respectively. By balancing the pressures of the two channels, a stable laminar flow can be obtained in the common central channel and if no sorting is performed all the cells are output from the top-right branch. When a cell is to be tested, light is sent through an optical waveguide facing the central channel, so that the cell is pushed into the "lower half" of the channel, and the pure buffer flux brings it to the constriction branch. After the selected cell blocks the constriction, a slow pressure ramp is applied, thanks to high-precision micropumps, from the input part until cell passes. The pressure values required to push cells through the constriction is defined as "passing pressure" and stored. In order to evaluate the possibility to use the "passing pressure" parameter to analyze cell mechanical properties, we performed different experiments on two pairs of cellular lines: tumorigenic (MCF7) and metastatic (MDA-MB231) human breast cancer cells and metastatic (A375P) and highly metastatic (A375MC2) human melanoma cells. The obtained results, shown in Fig.4a) and b), highlight that a statistically significant difference between the passing pressures of the considered populations is present.

Our results demonstrate that this constriction chip allows distinguishing cancer cells on the basis of their metastatic potential, which is positively correlated to the pressure required by cells to squeeze through the constriction.
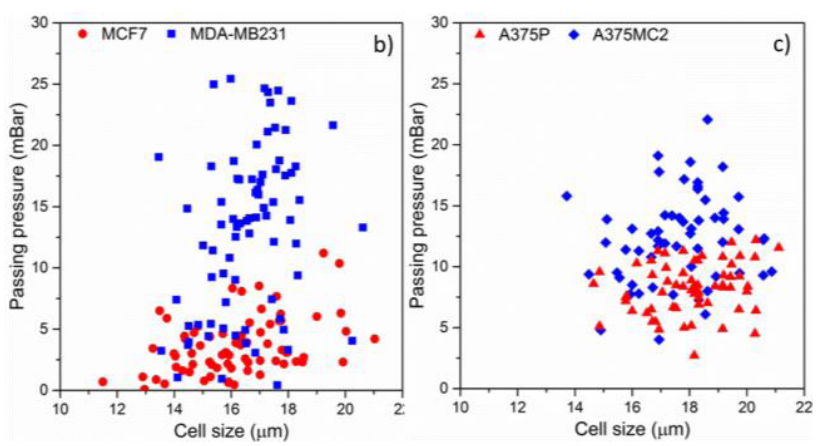

Figure 4. Passing pressure versus cell size of MCF7 and MDAMB231 (a) and A375P and A375MC2 cells (b) from the constriction chip.

Additionally, we investigated also the impact on cells of drugs able to affect microtubules organization and we observed significant changes in the passing-pressure distributions, thus suggesting that the proposed chip can even be applied for the analysis of drug treatments on single cells.

\section{Conclusions}

Femtosecond laser fabricated optofluidic devices allows probing mechanical properties for different purposes, ranging from health status analysis, drug screening or single cell sorting so as to collect cells for further analysis.

\section{References}

1. A. Fritsch, M. Höckel, T. Kiessling, K. D. Nnetu, F. Wetzel, M. Zink, and J. A. Käs, Nat. Phys. 6, 730 732 (2010).

2. S. Kumar, V.M. Weaver, Cancer Metastasis Reviews 28, 113-127 (2009).

3. F. Wottawah, S. Schinkinger, B. Lincoln, R. Ananthakrishnan, M. Romeyke, J. Guck and J. Käs, Phys. Rev. Lett. 94, 09103-4(2005)

4. J. El-Ali, P.K. Sorger and K.F. Jensen, Nature, 442, 403 (2006)

5. N. Bellini, F. Bragheri, I. Cristiani, J. Guck, R. Osellame and G. Whyte, Biomed. Opt. Express, 3, 2658-2668.(2012).

6. R. Osellame, H. J. W. M. Hoekstra, G. Cerullo, M. Pollnau, Laser \& Photonics Reviews 5, 442, (2011).

7. T. Yang, P. Paiè, G. Nava, F. Bragheri, R. Martinez Vazquez, P. Minzioni, M. Veglione, M. Di Tano, C. Mondello, R. Osellame and I. Cristiani, Lab on Chip 15, 1262-1266 (2015).

8. R. Martinez Vazquez, G. Nava, M. Veglione, T. Yang, F. Bragheri, P. Minzioni, E. Bianchi, M. Di Tano, I. Chiodi, R. Osellame, C. Mondello and I. Cristiani, Integrative Biology 7, 477-484 (2015).

9. F. Bragheri, P. Minzioni, R. Martinez Vazquez, N. Bellini, P. Paiè, C. Mondello, R. Ramponi, I. Cristiani and R. Osellame, Lab on Chip 12, 37793784 (2012).

10. T.Yang, G. Nava, P. Minzioni, M. Veglione, F. Bragheri, F.D. Lelii, R. Martinez Vazquez, R. 
MATEC Web of Conferences

Osellame and I. Cristiani, Biomed. Opt. Express 6,

236345 (2015). 